\title{
Untersuchung der Reduktion der harmonischen elektrischen Größen beim Betrieb einer Synchron-Reluktanzmaschine
}

\author{
M. Nikowitz, M. Hofer OVE, M. Schrödl OVE
}

In dieser Arbeit wird die Problematik der unerwünschten Harmonischen im Strangstrom sowie in der Strangspannung behandelt. Diese Harmonischen treten besonders im Fall der Synchron-Reluktanzmaschine mit Einzelpolen auf. Es wird das konventionelle mathematische Modell erweitert, um die Erzeugung der Harmonischen erklären und beschreiben zu können. Auf Grundlage des modifizierten mathematischen Modells wird eine Regler-Struktur vorgestellt, welche die unerwünschten Harmonischen im Strom bzw. in der Spannung erheblich reduziert. Dieses Regelkonzept benötigt lediglich die Frequenz der Harmonischen, wodurch ein universeller und robuster Einsatz möglich wird. Schlussendlich werden die vorgestellten Regelkonzepte anhand von Experimenten an einem Prototyp verifiziert.

Schlüsselwörter: Synchronmaschine; Reluktanzmaschine; Harmonische; elektrische Antriebe; Echtzeit-Kompensation

Analysing reduction possibilities of harmonic disturbances in synchronous reluctance machine drives.

In this paper a current and voltage ripple reduction method is evaluated. These harmonic disturbances occur especially in synchronous reluctance machines with salient pole configuration. The fundamental wave model has to be enhanced to handle these unwanted harmonic effects. With this extended model a current and voltage ripple suppression method was developed, which only needs the frequency of the harmonic disturbance. Finally, the introduced control algorithms were verified on the prototype.

Keywords: synchronous machine; reluctance machine; harmonics; electrical drive; real-time compensation

Eingegangen am 8. November 2018, angenommen am 21. Februar 2019, online publiziert am 12. März 2019

() The Author(s) 2019

\section{Einleitung}

Synchron-Reluktanzmaschinen (SynRM) haben eine immer größer werdende Bedeutung in der Industrie. Nicht zuletzt der höhere Wirkungsgrad verglichen mit herkömmlichen Asynchronmaschinen (ASM) im Teillast-Bereich bzw. bei geringer Drehzahl erklärt diesen Trend $[1-3,11]$. Ebenso die einfache und kostengünstige Produktion der Rotoren macht diesen Motortyp attraktiv für den breiten industriellen Einsatz und stellt eine wesentliche wirtschaftliche Konkurrenz zur hoch effizienten Permanentmagnet-erregten Synchronmaschine (PMSM) dar. Um den Wirkungsgrad der SynRM noch weiter zu erhöhen, wäre der Einsatz einer Permanentmagnet unterstützen SynRM denkbar [17]. Dies stellt einen Kompromiss zwischen der kostengünstigen SynRM ohne Permanentmagnete und der hoch effizienten PMSM dar. Ein weiterer wesentlicher Aspekt bei SynRM ist der mögliche lagegeberlose Betrieb [5, 6, 13]. Dieser reduziert zum einen die Anschaffungs- und Wartungskosten, zum anderen wird die Ausfallsicherheit erhöht. Reluktanzmaschinen haben allerdings, je nach Rotortyp, auch gewisse Nachteile. Die Maschine mit Flussbarrieren benötigt beispielsweise einen minimalen Sättigungsstrom um eine Achsigkeit zu erzeugen, welche zum Aufbau eines Drehmoments notwendig ist [8]. Diese minimale Achsigkeit wird auch für einen lagegeberlosen Betrieb benötigt, was ausführlich in [13] behandelt wurde. Eine Maschine mit ausgeprägten Polen hat dieses Problem beispielsweise nicht. Bei diesem Maschinentyp werden allerdings vermehrt harmonische Störgrößen generiert [4], welche sich negativ auf diverse Anwendungen auswirken können. Der Fokus dieser Arbeit liegt auf der gezielten Reduktion einer, durch die
Anwendung festgelegten, Störgröße einer Reluktanzmaschine mit ausgeprägten Polen.

\section{Mathematische Grundgleichungen der SynRM}

Die SynRM lässt sich am einfachsten im rotorfesten Koordinatensystem, dem dq-Koordinatensystem, beschreiben. Die d-Achse ist hierbei jene Achse, welche in die Richtung der kleinst möglichen Reluktanz weißt, wodurch die q-Achse, elektrisch orthogonal darauf stehend, in die Richtung des größten magnetischen Widerstands zeigt (siehe Abb. 1). Die Abweichung der d-Achse von der Symmetrieachse des ausgeprägten Pols kommt daher, dass der Winkel $\beta_{P}$ zwischen den einzelnen Polen nicht exakt $90^{\circ}$ beträgt [4]. Mittels räumlicher Fourierreihen-Entwicklung der magnetischen Leitwertsverteilung lässt sich über den Grundschwingungsanteil die d-Achse bestimmen.

Wie bereits in Abschn. 1 erwähnt, handelt es sich bei der untersuchten Maschine um eine SynRM mit ausgeprägten Polen [4]. Dieser Typ von Maschine weist praktisch keinerlei Sättigungseffekte in der q-Richtung auf, wodurch der Ansatz $l_{q}=$ konst. gerechtfer-

Nikowitz, Mario, Institut für Energiesysteme und Elektrische Antriebe, Technische Universität Wien, Gusshausstraße 25/370-2, 1040 Wien, Österreich

(E-Mail: mario.nikowitz@tuwien.ac.at); Hofer, Matthias, Institut für Energiesysteme und Elektrische Antriebe, Technische Universität Wien, Gusshausstraße 25/370-2, 1040 Wien, Österreich (E-Mail: matthias.hofer@tuwien.ac.at); Schrödl, Manfred, Institut für Energiesysteme und Elektrische Antriebe, Technische Universität Wien, Gusshausstraße 25/370-2, 1040 Wien, Österreich (E-Mail: manfred.schroedl@tuwien.ac.at) 


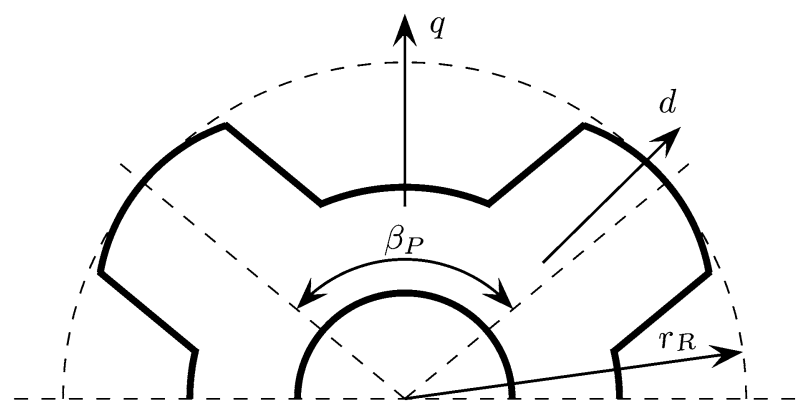

Abb. 1. Rotorblechschnitt der SynRM mit ausgeprägten Polen

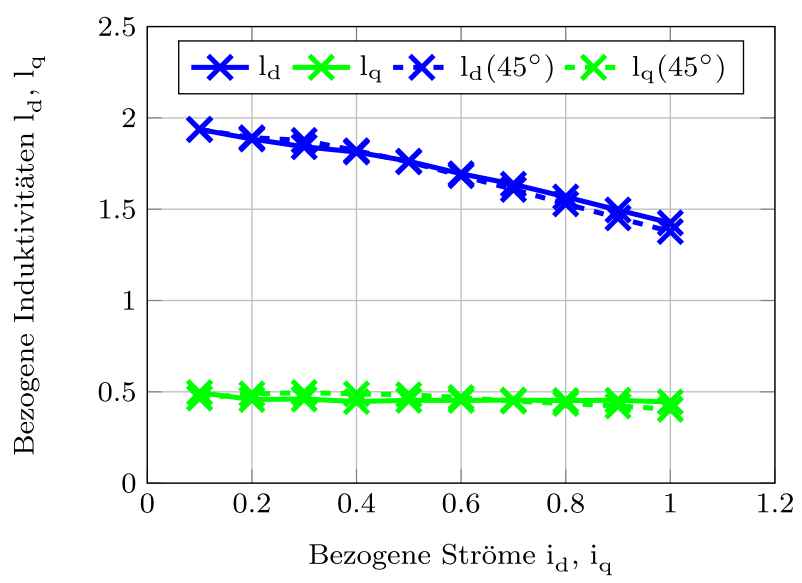

Abb. 2. Gemessene bezogene Induktivitäten $I_{d}$ und $I_{q}$ bzw. $I_{d}\left(45^{\circ}\right)$ und $I_{q}\left(45^{\circ}\right)$ als Funktion des jeweiligen Stromes $i_{d}$ und $i_{q}$

tigt ist. Bei der Längsinduktivität können Sättigungseffekte in guter Näherung als Funktion des Längsstroms $i_{d}$ berücksichtigt werden, d.h. es gilt $I_{d}=I_{d}\left(i_{d}\right)$. Es lassen sich folglich die GrundwellenFlussverkettungsgleichungen gemäß (2.1) und (2.2) aufstellen.

$$
\begin{aligned}
\psi_{d} & =I_{d}\left(i_{d}\right) i_{d} \\
\psi_{q} & =I_{q} i_{q}
\end{aligned}
$$

Hierbei repräsentiert $\psi=\psi_{d}+j \psi_{q}$ den komplexen, bezogenen Flussverkettungsraumzeiger, $\underline{i}=i_{d}+j i_{q}$ den komplexen, bezogenen Stromraumzeiger, $I_{d}$ die bezogene Längsinduktivität und $I_{q}$ die bezogene Querinduktivität.

Abbildung 2 stellt den Verlauf der gemessenen Induktivitäten $I_{d}$ und $I_{q}$ bzw. $I_{d}\left(45^{\circ}\right)$ und $I_{q}\left(45^{\circ}\right)$ als Funktion des jeweiligen Stromes $i_{d}$ und $i_{q}$ dar. Die Messung von $I_{d}\left(i_{d}\right)$ und $l_{q}\left(i_{q}\right)$ wurden mittels reinem q-Strom $i_{q}$ bzw. reinem d-Strom $i_{d}$ durch geführt. Dies hat den Effekt, dass die magnetische Kopplung zwischen der $d$ - und der q-Achse keine Rolle spielt (siehe Gl. (4.11) und (4.12)). Analog dazu wurden die Induktivitäten $I_{d}\left(45^{\circ}\right)$ und $I_{q}\left(45^{\circ}\right)$ mittels eines Stromraumzeigers gemessen, welcher $45^{\circ} \mathrm{im}$ dq-Koordinatensystem steht. Hierbei würde eine Querkopplung ins Gewicht fallen, da sowohl die d-Stromkomponente als auch die qStromkomponente ungleich 0 wären. Aus Abb. 2 ist ersichtlich, dass kaum ein Unterschied zwischen der Messung von $I_{d}$ und $I_{d}\left(45^{\circ}\right)$ bzw. $I_{q}$ und $I_{q}\left(45^{\circ}\right)$ erkennbar ist. In anderen Worten bedeutet dies, dass die magnetische Kopplung zwischen der $\mathrm{d}$ - und der q-Achse vernachlässigbar klein ist und der vereinfachte Ansatz (2.1) und (2.2) gerechtfertigt ist.

Der komplexe, bezogene Statorspannungsraumzeiger $\underline{u}=u_{d}+$ $j u_{q}$ lässt sich mit (2.1) und (2.2) in einfachster Form über die verkop- pelten Differentialgleichungen (2.3) und (2.4) beschreiben.

$$
\begin{aligned}
& u_{d}=r i_{d}+I_{d}\left(i_{d}\right) \frac{d i_{d}}{d \tau}-\omega_{m} I_{q} i_{q} \\
& u_{q}=r i_{q}+I_{q} \frac{d i_{q}}{d \tau}+\omega_{m} l_{d}\left(i_{d}\right) i_{d}
\end{aligned}
$$

Hierbei repräsentiert $r$ den bezogenen Statorwiderstand und $\omega_{m}$ die bezogene, elektrische Kreisfrequenz. Schlussendlich kann man aus den bisherigen Gleichungen auf die Gleichung für das bezogene Drehmoment $m$ (2.5) schließen.

$$
m=-\operatorname{Im}\left\{\underline{\psi} \underline{i}^{*}\right\}=\left(I_{d}\left(i_{d}\right)-I_{q}\right) i_{d} i_{q}
$$

\section{Darstellung der Problematik}

Durch die Wahl der Rotorgeometrie kann man gezielt diverse Betriebsverhalten einer SynRM optimieren. Beispielsweise kann durch die Wahl einer Maschine mit ausgeprägten Polen der Wirkungsgrad im Vergleich zur Maschine mit Flussbarriere-Rotor erhöht werden [7]. Dies hat allerdings den Nachteil, dass die ungewollten harmonische Größen deutlich stärker ausfallen [4, 8]. Das Ausmaß des Auftretens der Harmonischen wird durch folgende Messungen an der Maschine im herkömmlichen feldorientierten, sensorbehafteten Betrieb analysiert.

Um diverse Koordinatentransformationen und mathematische Aufbereitungen aus der Messung herauszuhalten, wurde für die Analyse der gemessene Strom bzw. die Spannung der Phase U herangezogen. Diese Größen entsprechen, aufgrund der Sternschaltung der Maschine, dem Strom $i_{\alpha}$ und der Spannung $u_{\alpha}$. Das Spektrum des Stromes $i_{\alpha}$ (siehe Abb. 3) und der Spannung $u_{\alpha}$ (siehe Abb. 4) wird als Funktion des Betrags des bezogenen Stromraumzeigers $\left|i_{-\alpha \beta}\right|$ und der elektrischen Rotorwinkelgeschwindigkeit $\omega_{m}$ dargestellt.

Aus Abb. 3 und Abb. 4 ist ersichtlich, dass die dominanten harmonischen Rippel bei der 11-ten und 13-ten sowie bei der 5-ten und 7-ten Harmonischen liegen. Dieser Effekt der Erzeugung der harmonischen Störgrößen lässt sich nicht mit dem im Kap. 2 angeführten Modell beschreiben, wodurch das Modell erweitert werden muss.

\section{Erweitertes mathematisches Modell}

Ein Ansatz zur Lösung des Problems ist die Erweiterung der Längsund Querinduktivität um eine winkelabhängige Komponente. Der Vollständigkeit halber wird anfänglich das Grundschwinungsmodell noch mit gerechnet, wobei im späteren Verlauf der Herleitung der Fokus auf dem Oberschwingungsmodell liegt. Die zuvor erwähnte Winkelabhängigkeit kann in erster Näherung, bei konstanter Drehzahl, in folgende Zeitabhängigkeit übersetzt werden.

$$
\begin{aligned}
& I_{d}(\tau)=I_{d, 0}+I_{d, k}(\tau) \\
& I_{q}(\tau)=I_{q, 0}+I_{q, k}(\tau)
\end{aligned}
$$

$I_{d, 0}$ und $I_{q, 0}$ stellen die zeitunabhängigen Anteile gemäß Kap. 2 dar und $I_{d, k}(\tau)$ und $I_{q, k}(\tau)$ werden zur Modellierung der k-ten harmonischen Effekte herangezogen. Ebenso lässt sich der komplexe bezogene Strom- und Spannungsraumzeiger in einen Grundschwingungsanteil und einen Anteil der k-ten Harmonischen aufteilen.

$$
\begin{aligned}
& u_{d}(\tau)=u_{d, 0}+u_{d, k}(\tau) \\
& u_{q}(\tau)=u_{q, 0}+u_{q, k}(\tau) \\
& i_{d}(\tau)=i_{d, 0}+i_{d, k}(\tau) \\
& i_{q}(\tau)=i_{q, 0}+i_{q, k}(\tau)
\end{aligned}
$$



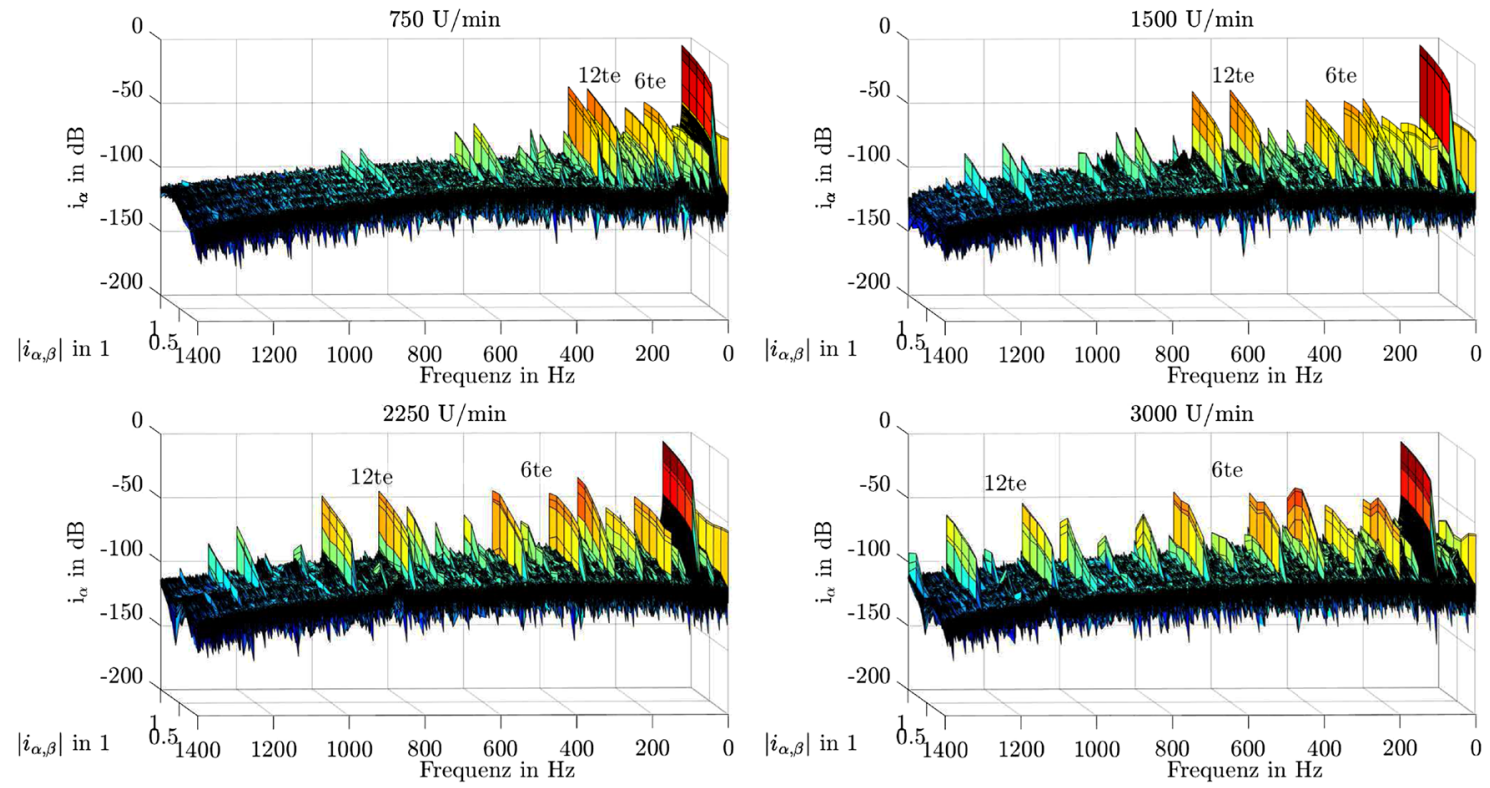

Abb. 3. Strom-Rippel als Funktion der Frequenz $\omega_{m}$ und des Betrags des Statorstromraumzeigers $\left|i_{\alpha, \beta}\right|$
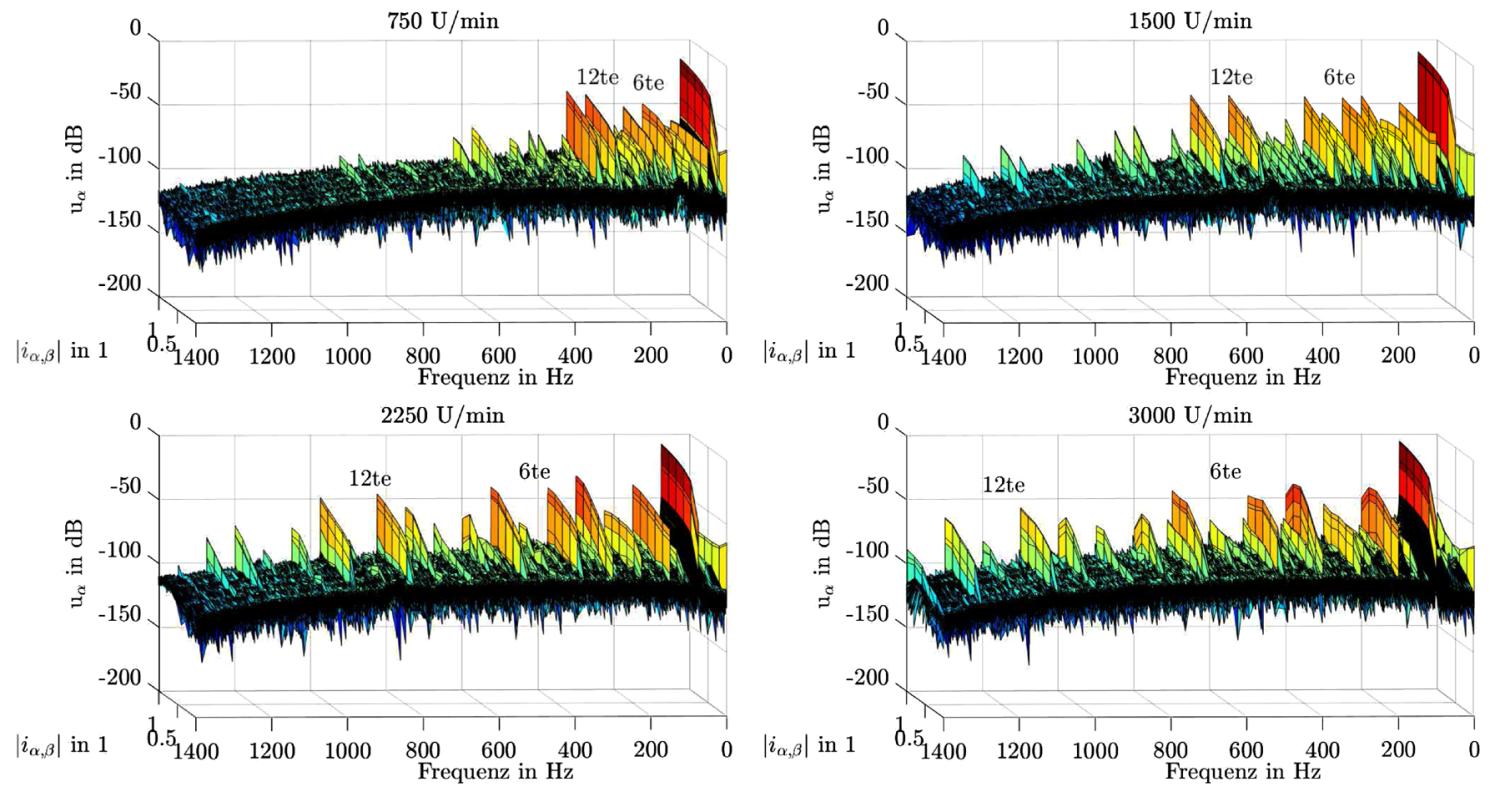

Abb. 4. Spannungs-Rippel als Funktion der Frequenz $\omega_{m}$ und des Betrags des Statorstromraumzeigers $\left|\underline{i}_{\alpha, \beta}\right|$

Durch die zeitlich veränderlichen Induktivitäten muss die Gleichung für den Statorspannungsraumzeiger ebenfalls adaptiert werden, was zu den Gl. (4.7) und (4.8) führt.

$$
\begin{aligned}
u_{d} & =r i_{d}+\frac{d \psi_{d}}{d \tau}-\omega_{m} l_{q} i_{q} \\
& =r i_{d}+\frac{d l_{d}\left(i_{d}\right)}{d \tau} i_{d}+l_{d}\left(i_{d}\right) \frac{d i_{d}}{d \tau}-\omega_{m} l_{q} i_{q} \\
u_{q} & =r i_{q}+\frac{d \psi_{q}}{d \tau}+\omega_{m} l_{d}\left(i_{d}\right) i_{d} \\
& =r i_{q}+\frac{d l_{q}}{d \tau} i_{q}+l_{q} \frac{d i_{q}}{d \tau}+\omega_{m} l_{d}\left(i_{d}\right) i_{d}
\end{aligned}
$$

Um das System möglichst einfach zu halten, wurde die Stromabhängigkeit der Längsinduktivität unabhängig vom Rippelstrom $i_{d, k}$ gewählt. Es gilt somit näherungsweise $I_{d}\left(i_{d}\right) \approx I_{d}\left(i_{d, 0}\right)=I_{d}$.

Unter Verwendung von GI. (4.3)-(4.8) und der Annahme konstanter Drehzahl lässt sich die Statorspannungsgleichung der $d$-Richtung (4.9) und q-Richtung (4.10) wie folgt angeben.

$$
\begin{aligned}
u_{d, 0}+u_{d, k}(\tau)= & r\left(i_{d, 0}+i_{d, k}\right)+\frac{d l_{d, k}}{d \tau}\left(i_{d, 0}+i_{d, k}\right) \\
& +\left(I_{d, 0}+I_{d, k}\right) \frac{d i_{d, k}}{d \tau}-\omega_{m}\left(I_{q, 0}+I_{q, k}\right)\left(i_{q, 0}+i_{q, k}\right)
\end{aligned}
$$




$$
\begin{aligned}
u_{q, 0}+u_{q, k}(\tau)= & r\left(i_{q, 0}+i_{q, k}\right)+\frac{d l_{q, k}}{d \tau}\left(i_{q, 0}+i_{q, k}\right) \\
& +\left(I_{q, 0}+I_{q, k}\right) \frac{d i_{q, k}}{d \tau}+\omega_{m}\left(I_{d, 0}+I_{d, k}\right)\left(i_{d, 0}+i_{d, k}\right)
\end{aligned}
$$

Durch Separation und Umformung der Grundschwingungsanteile aus Gl. (4.9) und (4.10) erhält man eine Gleichung für die Grundschwingungsinduktivität in d-Richtung (4.11) und q-Richtung (4.12).

$$
\begin{aligned}
& I_{d, 0}=\frac{u_{q, 0}-r i_{q, 0}}{\omega_{m} i_{d, 0}} \\
& l_{q, 0}=-\frac{u_{d, 0}-r i_{d, 0}}{\omega_{m} i_{q, 0}}
\end{aligned}
$$

Aus den übrigen Termen lässt sich ein nichtlineares Differentialgleichungssystem (4.13) und (4.14) aufstellen, welches sich durch folgende Linearisierung um den Arbeitspunkt vereinfachen lässt.

$$
\begin{aligned}
0= & \left(i_{d, 0}+i_{d, k}\right) \cdot \frac{d l_{d, k}}{d \tau}+\left(\frac{d i_{d, k}}{d \tau}\right) \cdot l_{d, k} \\
& -\left(\omega_{m}\left(i_{q, 0}+i_{q, k}\right)\right) \cdot l_{q, k} \\
& +\left(r i_{d, k}-u_{d, k}+l_{d, 0} \frac{d i_{d, k}}{d \tau}-\omega_{m} l_{q, 0} i_{q, k}\right) \\
\approx & i_{d, 0} \cdot \frac{d l_{d, k}}{d \tau}+\frac{d i_{d, k}}{d \tau} \cdot l_{d, 0}-\omega_{m} i_{q, 0} \cdot l_{q, k} \\
& +r i_{d, k}-u_{d, k}-\omega_{m} l_{q, 0} i_{q, k} \\
0= & \left(i_{q, 0}+i_{q, k}\right) \cdot \frac{d l_{q, k}}{d \tau}+\left(\frac{d i_{q, k}}{d \tau}\right) \cdot l_{q, k} \\
& +\left(\omega_{m}\left(i_{d, 0}+i_{d, k}\right)\right) \cdot l_{d, k} \\
& +\left(r i_{q, k}-u_{q, k}+l_{q, 0} \frac{d i_{q, k}}{d \tau}+\omega_{m} l_{d, 0} i_{d, k}\right) \\
\approx & i_{q, 0} \cdot \frac{d l_{q, k}}{d \tau}+\frac{d i_{q, k}}{d \tau} \cdot l_{q, 0}+\omega_{m} i_{d, 0} \cdot l_{d, k} \\
& +r i_{q, k}-u_{q, k}+\omega_{m} l_{d, 0} i_{d, k}
\end{aligned}
$$

Durch diese Vereinfachung kann das nichtlineare Differentialgleichungssystem in ein lineares überführt werden, wodurch die Anwendung der Laplace-Transformation (4.15) und (4.16) möglich ist. Die Laplace-Transformation ermöglicht die Einführung der Rippel-

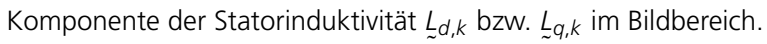

$$
\begin{aligned}
& {\underset{\sim}{d, k}}_{v}-r l_{d, k}+\omega_{m} l_{q, 0} I_{\sim, k}-s l_{d, 0} I_{d, k} \\
& =s L_{\sim}, k i_{d, 0}-\omega_{m} L_{\sim}, k i_{q, 0} \\
& \bigcup_{\sim q, k}-r l_{\sim, k}-\omega_{m} l_{d, 0} l_{\sim}, k-s l_{q, 0} l_{\sim, k} \\
& =s L_{q, k} i_{q, 0}+\omega_{m} L_{d, k} i_{d, 0}
\end{aligned}
$$

Durch Umformung auf die gesuchten Induktivitäten $L_{d, k}$ und $L_{q, k}$ erhält man die GI. (4.17) und (4.18) im Bildbereich.

$$
\begin{aligned}
& \underline{\sim}_{d, k}=\frac{1}{i_{d, 0}}\left[\frac{s}{s^{2}+\omega_{m}^{2}}\left({\underset{\sim}{d, k}}_{U^{\prime}}-r l_{d, k}\right)\right. \\
& \left.+\frac{\omega_{m}}{s^{2}+\omega_{m}^{2}}\left({\underset{\sim}{q, k}}_{U^{\prime}}-r I_{q, k}\right)-I_{d, 0} l_{d, k}\right] \\
& \stackrel{L}{q, k}_{q, k}=\frac{1}{i_{q, 0}}\left[-\frac{\omega_{m}}{s^{2}+\omega_{m}^{2}}\left({\underset{\sim}{d, k}}_{d, k}-r l_{d, k}\right)\right. \\
& \left.+\frac{s}{s^{2}+\omega_{m}^{2}}\left(\cup_{\sim, k}+r l_{\sim, k}\right)-l_{q, 0_{\sim}} l_{q, k}\right]
\end{aligned}
$$

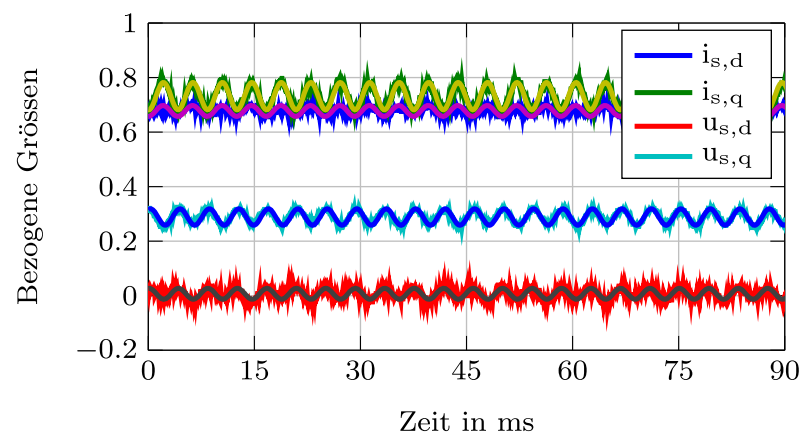

Abb. 5. Messung der bezogenen Statorspannung und des bezogenen Strangstroms im dq-Koordinatensystem im stromgeregelten Betrieb bei $600 \mathrm{U} \mathrm{min}^{-1}$

Zur Modellierung des Rippelstroms $i_{d, k}$ und $i_{q, k}$ und der Rippelspannung $u_{d, k}$ und $u_{q, k}$ wird die Messung, dargestellt in Abb. 5, herangezogen.

Der bezogenen Rippelstrom- $\underline{i}_{k}=i_{d, k}+j i_{q, k}$ und Rippelspannungsraumzeiger $\underline{u}_{k}=u_{d, k}+j u_{q, k}$ lassen sich gemäß GI. (4.19) bis (4.22) bestimmen.

$$
\begin{aligned}
& i_{d}=i_{d, 0}+\hat{i}_{d, k} \cos \left(k \omega_{m} \tau\right) \\
& i_{q}=i_{q, 0}+\hat{i}_{q, k} \cos \left(k \omega_{m} \tau\right) \\
& u_{d}=u_{d, 0}-\hat{u}_{d, k} \cos \left(k \omega_{m} \tau\right) \\
& u_{q}=u_{q, 0}-\hat{u}_{q, k} \cos \left(k \omega_{m} \tau\right)
\end{aligned}
$$

Gleichungen (4.19) bis (4.22) Laplace transformiert, in Gl. (4.17) und (4.18) eingesetzt und Partialbruch zerlegt ergibt den Rippelanteil der Induktivitäten im Bildbereich (4.23) und (4.24).

$$
\begin{aligned}
\underline{L}_{d, k}= & \frac{1}{i_{d, 0}}\left[\frac{k^{2}}{k^{2}-1} \cdot \frac{\hat{u}_{d, k}+r \hat{i}_{d, k}}{k \omega_{m}} \cdot \frac{k \omega_{m}}{s^{2}+\left(k \omega_{m}\right)^{2}}\right. \\
& +\frac{1}{k^{2}-1} \cdot \frac{\hat{u}_{d, k}+r \hat{i}_{d, k}}{\omega_{m}} \cdot \frac{\omega_{m}}{s^{2}+\omega_{m}^{2}} \\
& -\frac{1}{k^{2}-1} \cdot \frac{\hat{u}_{q, k}+r \hat{i}_{q, k}}{\omega_{m}} \cdot \frac{s}{s^{2}+\left(k \omega_{m}\right)^{2}} \\
& +\frac{1}{k^{2}-1} \cdot \frac{\hat{u}_{q, k}+\hat{i}_{q, k}}{\omega_{m}} \cdot \frac{s}{s^{2}+\omega_{m}^{2}} \\
& \left.+l_{d, 0} \hat{i}_{d, k} \cdot \frac{s}{s^{2}+\left(k \omega_{m}\right)^{2}}\right] \\
\underline{L}_{q, k}= & \frac{1}{i_{q, 0}}\left[\frac{k^{2}}{k^{2}-1} \cdot \frac{\hat{u}_{q, k}+r \hat{i}_{q, k}}{k \omega_{m}} \cdot \frac{k \omega_{m}}{s^{2}+\left(k \omega_{m}\right)^{2}}\right. \\
& +\frac{1}{k^{2}-1} \cdot \frac{\hat{u}_{q, k}+r \hat{i}_{q, k}}{\omega_{m}} \cdot \frac{\omega_{m}}{s^{2}+\omega_{m}^{2}} \\
& +\frac{1}{k^{2}-1} \cdot \frac{\hat{u}_{d, k}+r \hat{i}_{d, k}}{\omega_{m}} \cdot \frac{s}{s^{2}+\left(k \omega_{m}\right)^{2}} \\
& -\frac{1}{k^{2}-1} \cdot \frac{\hat{u}_{d, k}+r \hat{i}_{d, k}}{\omega_{m}} \cdot \frac{s}{s^{2}+\omega_{m}^{2}} \\
& \left.+l_{q, 0} \hat{i}_{q, k} \cdot \frac{s}{s^{2}+\left(k \omega_{m}\right)^{2}}\right]
\end{aligned}
$$

Nach der Rücktransformation ergibt sich für die zwölfte Harmonische $k=12$, d.h. $k^{2} \gg 1$, die vereinfachten Induktivitäten zu (4.25) 


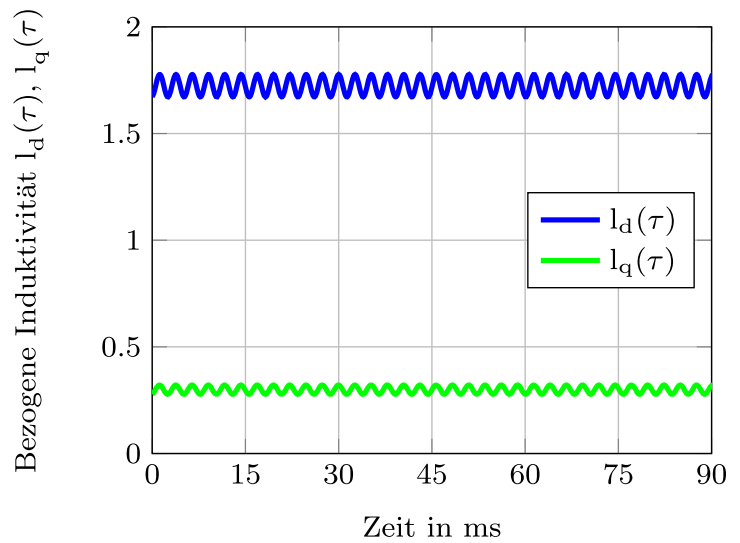

Abb. 6. Berechnung des Verlaufs von $I_{d}(\tau)$ und $I_{q}(\tau)$ bei $600 \mathrm{U} \mathrm{min}^{-1}$

und (4.26)

$$
\begin{aligned}
& I_{d, k} \approx \frac{\hat{u}_{d, k}+r \hat{i}_{d, k}}{k \omega_{m} \frac{1}{i_{d, 0}}} \cdot \sin \left(k \omega_{m} \tau\right)+I_{d, 0} \frac{\hat{i}_{d, k}}{i_{d, 0}} \cdot \cos \left(k \omega_{m} \tau\right) \\
& I_{q, k} \approx \frac{\hat{u}_{q, k}+r \hat{i}_{q, k}}{k \omega_{m} \frac{1}{i_{q, 0}}} \cdot \sin \left(k \omega_{m} \tau\right)+I_{q, 0} \frac{\hat{i}_{q, k}}{i_{q, 0}} \cdot \cos \left(k \omega_{m} \tau\right)
\end{aligned}
$$

Diese zuvor getroffene Vereinfachung würde beispielsweise im Fall der sechsten Harmonischen $k=6$ zwar einen etwas größeren Fehler nach sich ziehen als für $k=12$. Die so entstandenen $A b-$ weichungen des Modells zur Realität wären allerdings immer noch hinreichend gering und können vernachlässigt werden.

Abbildung 6 zeigt die mittels $\mathrm{Gl}$. (4.25) und (4.26), sowie den Messungen (4.19) bis (4.22) bestimmten Induktivitäten $I_{d}(\tau)$ und $I_{q}(\tau)$. Dies zeigt, dass der anfänglich getroffene Ansatz einer winkelbzw. (bei konstanter Drehzahl) zeitabhängiger Induktivität gerechtfertigt ist. Die Unterschiede der Amplitude der Oberschwinungsinduktivitäten $I_{d, k}$ und $I_{q, k}$ lässt sich auf die Unterschiedlich stark ausgeprägten Luftspalte der $\mathrm{d}$ - und der q-Richtung zurückführen (siehe Abb. 1)

\section{Regelkonzept}

Die in Kap. 3 auftretenden Harmonischen lassen sich nur durch eine Regelung unterdrücken, welche explizit die auftretenden Harmonischen berücksichtigt. Hierbei muss man grundsätzlich unterscheiden zwischen Rippelgrößen, die durch die PWM erzeugt werden [12] und Rippelgrößen, die durch die Maschinengeometrie hervorgerufen werden. Bei der vorliegenden Arbeit handelt es sich um den zweiten Fall, damit würde die naheliegendste Lösung sein, das Maschinendesign auf einen möglichst geringen Anteil an Oberschwin- gungen hin zu optimieren. Diese Optimierung wird allerdings durch Einbußen in der Performance erkauft [8]

Alternativ bzw. zusätzlich dazu kann die harmonische Störung im Sinne der Störgrößen-Vorsteuerung aufgeschaltet werden. Hier bleibt jedoch noch zu klären, wie die Störgröße gemessen bzw. bestimmt werden kann. Eine Variante ist die iterative Bestimmung der aufzuschalteten Größe, was allerdings einen Verzicht auf Determinismus nach sich zieht [10]. Eine weitere Variante ist das Anlegen eines Referenzsignals, welches empirisch oder mittels exakter motorspezifischer Modellierung offline ermittelt und in einer Tabelle abgespeichert wird [14, 16]. Nachteilig hierbei ist die Verringerung der Robustheit der Regelung bezüglich Parameterschwankungen bzw. der große Aufwand der notwendigen Anpassungen an einen Motortyp.

Die in dieser Arbeit vorgestellte Methode der Bestimmung der Störgröße basiert auf dem Prinzip des Synchrondemodulators [9]. Sie hat den Vorteil, dass lediglich die Ordnung der störenden Harmonischen und die aktuelle Geschwindigkeit bzw. Frequenz bekannt sein muss. Dies macht das Verfahren universell einsetzbar und robust gegen Parameterschwankungen der Maschine. Abbildung 7 zeigt das Blockschaltbild des Synchrondemodulators $\underset{\sim}{N}$, welcher über Gleichung (5.1) beschrieben werden kann.

$$
\begin{aligned}
& c(\tau)=\left(\begin{array}{ll}
\sin \left(k \omega_{m} \tau\right) & \cos \left(k \omega_{m} \tau\right)
\end{array}\right) \cdot\left(\begin{array}{cc}
\cos (\alpha) & -\sin (\alpha) \\
\sin (\alpha) & \cos (\alpha)
\end{array}\right) \\
& \cdot \varepsilon \int\left(\begin{array}{c}
\sin \left(k \omega_{m} \tau\right) \\
\cos \left(k \omega_{m} \tau\right)
\end{array}\right) w(\tau) d \tau
\end{aligned}
$$

Mittels Laplace Transformation lässt sich die Übertragungsfunktion $N_{\sim}^{N}(s)$ des phasenunabhängigen Synchrondemodulators, wie in (5.2) angegeben, bestimmen.

$$
N(s)=\varepsilon \cdot \frac{s \cos (\alpha)-k \omega_{m} \sin (\alpha)}{s^{2}+\left(k \omega_{m}\right)^{2}}
$$

Abbildung 8 und 9 zeigen die Auswirkung der Variablen $\varepsilon$ und $\alpha$ auf den Betragsfrequenzgang des Filters. $\varepsilon$ definiert die Schärfe des Bandpassfilters, wobei $\alpha$ als Tuning-Parameter zur Stabilitätsanalyse herangezogen werden kann.

Es ist aus dem Betragsfrequenzgang ersichtlich, dass ein bestimmter Frequenzbereich aus dem Spektrum herausgefiltert wird, welcher anschließend entsprechend der Störgrößen-Aufschaltung auf das System wirkt. Die konkreten Aufschaltungsvarianten werden näher in Kap. 6 und 7 erläutert.

Es wird ein Dämpfungsgrad gemäß (5.3) definiert:

$$
\xi=\frac{-\kappa}{\sqrt{\kappa^{2}+\mu^{2}}}, \quad \text { wobei } \lambda_{i}=\kappa \pm j \mu
$$

Bezüglich der Stabilität des Reglerkonzepts wurde das in Kap. 4 vorgestellte erweiterte Modell linearisiert und der Dämpfungsgrad $\xi$

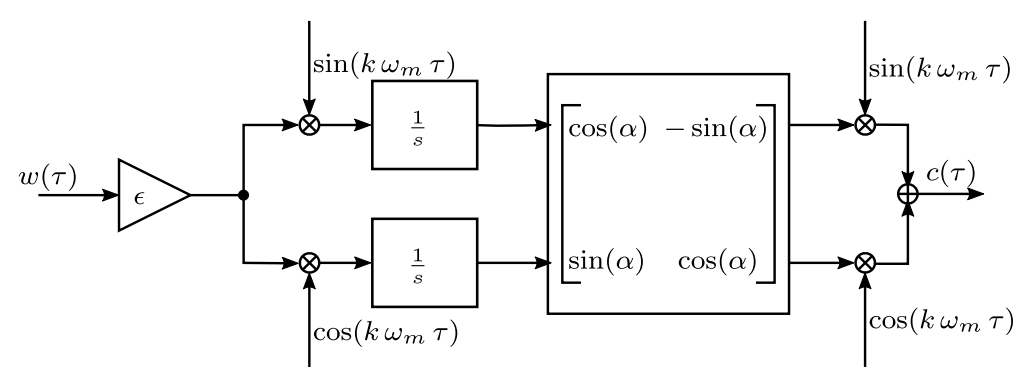

Abb. 7. Phasenunabhängiger Synchrondemodulator, Eingang $w(\tau)$, Ausgang $c(\tau)$ 


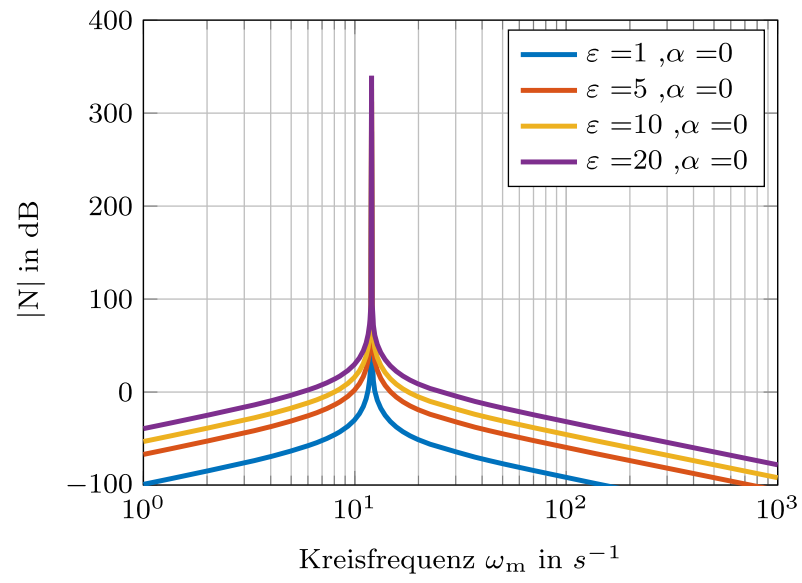

Abb. 8. Betragsfrequenzgang $|\underset{\sim}{N(s)}|$ als Funktion von $\varepsilon$

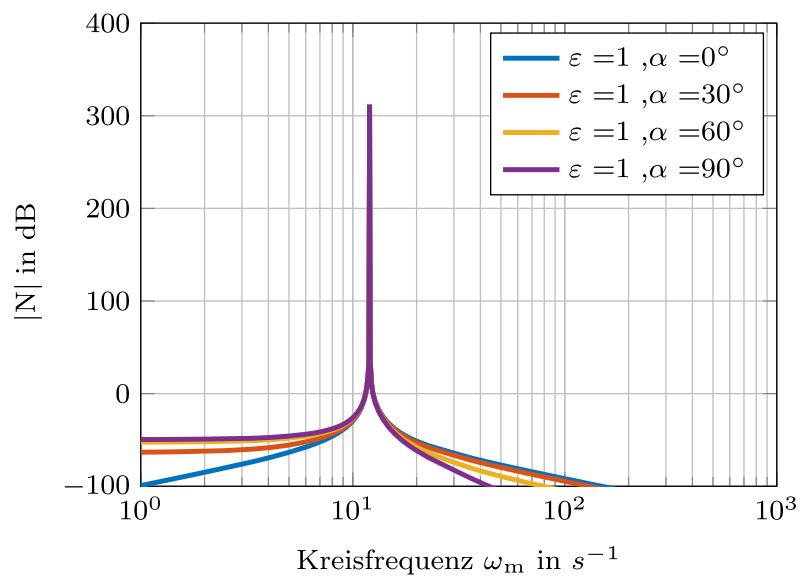

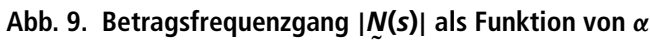

(5.3) der konjugiert komplexen Nullstelle $\lambda_{i}$ als Maß der Stabilität herangezogen.

\section{Analyse des Rippels des Strangstroms}

Den nachstehenden Messungen ist der folgende Messaufbau zugrunde gelegt. Der Motor wird mit einem Spannungszwischenkreisumrichter mit einer PWM-Spannung (16 kHz) gespeist, welcher mittels galvanisch getrennten Stromwandlern den ist-Zustand der Phasenströme misst. Für die benötigen Phasenspannungen werden die Soll-Spannungen des Stromreglers herangezogen.

Um den Rippel im Strangstrom zu reduzieren, wird sowohl für die d-Richtung als auch für die q-Richtung ein separates Filter $N_{d}$ bzw. $N_{q}$ verwendet. Abbildung 10 zeigt strichliert gezeichnet die verwendete Reglerstruktur.

Beispielsweise für die d-Richtung wird die Regelabweichung $i_{d, \text { soll }}-i_{d, \text { ist }}$ dem Filter $N_{d}$ zugeführt, welches die k-te Harmonische phasenrichtig herausfiltert. Diese bestimmte Störgröße wird anschließend direkt auf die Soll-Spannung $u_{d, \text { soll }}$ aufgeschaltet, was die Reduktion des Stromrippels in der d-Richtung nach sich zieht. Analoges gilt ebenfalls für die q-Strom Komponente.

Für die Stabilitäts-Analyse wird, wie in Abschn. 5 erwähnt, das in Abb. 10 dargestellte System linearisiert und die Stabilität mittels des Dämpfungsgrads $\xi_{d}$ bzw. $\xi_{q}$ bewertet (siehe Abb. 11). Es ist somit ersichtlich, dass sich der optimale Parametersatz für eine robuste

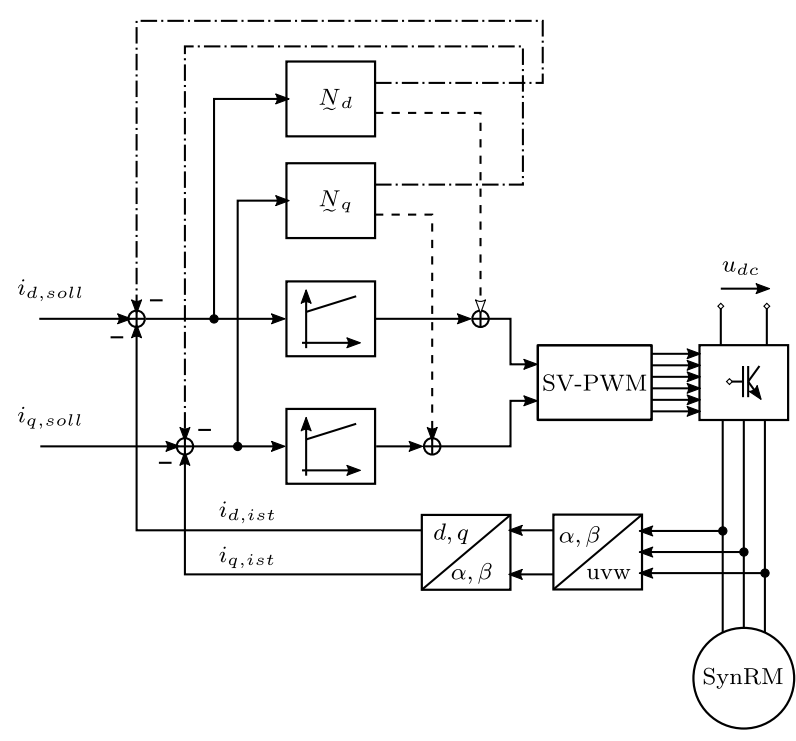

Abb. 10. Reglerstruktur zur Reduktion des Stromrippels (strichliert) bzw. des Spannungsrippels (strich-punktiert)

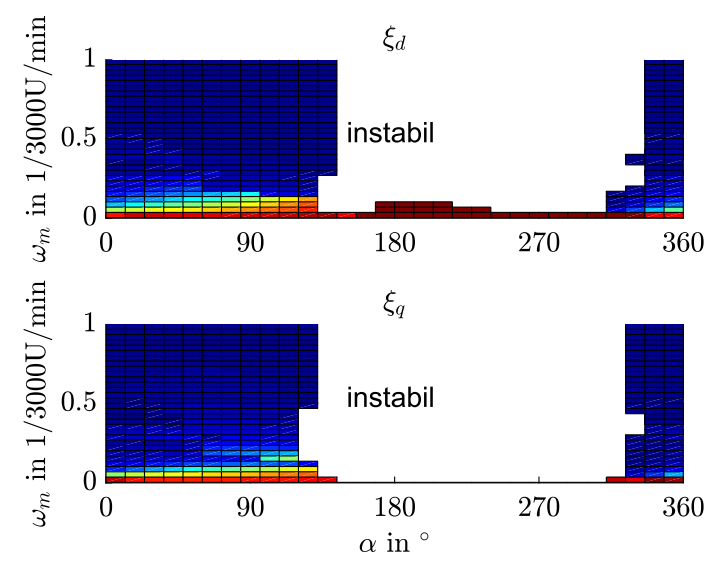

Abb. 11. Stromrippel Reduktion: Dämpfungsgrad $\xi_{d}$ und $\xi_{q}$ als Funktion von $\alpha$ und der bezogenen Drehzahl $\omega_{m}$

Stromrippel-Reduktion zu (6.1) ergibt.

$$
\begin{aligned}
& \alpha_{\text {Strom }}=90^{\circ} \\
& \varepsilon_{\text {Strom }} \propto \omega_{m}
\end{aligned}
$$

Abbildung 12 veranschaulicht die Reduktion des Stromrippels, wobei aus Tab. 1 die gemessene Reduktion des Stromrippels bei unterschiedlichen Drehzahlen zu entnehmen ist. Die Auswirkungen des Kompensationsalgorithmuses auf die anderen verbleibenden Harmonischen im Stromrippel sind hierbei vernachlässigbar gering

Durch die Reduktion des Stromrippels lassen sich die Verluste der Maschine deutlich reduzieren [15].

\section{Analyse des Rippels der Strangspannung}

Analog zur Rippelstrom-Reduktion in Abschn. 6 wird auch im Fall der Spannungsrippel-Reduktion die d- als auch die q-Richtung einzeln behandelt. Die Regler-Struktur ist aus Abb. 10 strich-punktiert gezeichnet zu entnehmen.

Im Fall der d-Richtung wird die Regelabweichung $i_{d, \text { soll }}-i_{d, \text { ist }}$ dem Filter $N_{d}$ zugeführt, welches die k-te Harmonische phasenrichtig her- 
Tab. 1. Gemessene Verstärkung der Rippelgrößen nach Anwendung der Störgrößen-Aufschaltung zur Stromrippel Reduktion

\begin{tabular}{lllll}
\hline Drehzahl & $\begin{array}{l}\text { Ordnung der } \\
\text { Harmonischen }\end{array}$ & $\begin{array}{l}\text { bezogener Betrag } \\
\text { Statorstromraumzeiger }\end{array}$ & $\begin{array}{l}\text { Veränderung } \\
\text { Statorstromrippel }\end{array}$ & $\begin{array}{l}\text { Veränderung } \\
\text { Statorspannungsrippel }\end{array}$ \\
\hline $750 \cup \min ^{-1}$ & 11 & $\left|i_{\alpha \beta}\right|=1$ & $-37,6 \mathrm{~dB}$ & $6,3 \mathrm{~dB}$ \\
& 13 & $\left|i_{\alpha \beta}\right|=1$ & $-43,0 \mathrm{~dB}$ & $3,0 \mathrm{~dB}$ \\
$1500 \cup \min ^{-1}$ & 11 & $\left|i_{\alpha \beta}\right|=1$ & $-37,5 \mathrm{~dB}$ & $10,3 \mathrm{~dB}$ \\
& 13 & $\left|i_{\alpha \beta}\right|=1$ & $-37,6 \mathrm{~dB}$ & $7,4 \mathrm{~dB}$ \\
$2250 \cup \min ^{-1}$ & 11 & $\left|i_{\alpha \beta}\right|=1$ & $-45,2 \mathrm{~dB}$ & $11,2 \mathrm{~dB}$ \\
& 13 & $\left|i_{\alpha \beta}\right|=1$ & $-39,8 \mathrm{~dB}$ & $14,4 \mathrm{~dB}$ \\
$3000 \cup \min ^{-1}$ & 11 & $\left|i_{\alpha \beta}\right|=1$ & $-40,8 \mathrm{~dB}$ & $10,2 \mathrm{~dB}$ \\
& 13 & $\left|i_{\alpha \beta}\right|=1$ & $-46,1 \mathrm{~dB}$ & $9,2 \mathrm{~dB}$ \\
\hline
\end{tabular}

Tab. 2. Gemessene Verstärkung der Rippelgrößen nach Anwendung der Störgrößen-Aufschaltung zur Spannungsrippel Reduktion

\begin{tabular}{lllll}
\hline Drehzahl & $\begin{array}{l}\text { Ordnung der } \\
\text { Harmonischen }\end{array}$ & $\begin{array}{l}\text { bezogener Betrag } \\
\text { Statorstromraumzeiger }\end{array}$ & $\begin{array}{l}\text { Veränderung } \\
\text { Statorstromrippel }\end{array}$ & $\begin{array}{l}\text { Veränderung } \\
\text { Statorspannungsrippel }\end{array}$ \\
\hline $750 \mathrm{U} \mathrm{min}^{-1}$ & 11 & $\left|i_{\alpha \beta}\right|=1$ & $-1,7 \mathrm{~dB}$ & $-13,3 \mathrm{~dB}$ \\
& 13 & $\left|i_{\alpha \beta}\right|=1$ & $-1,2 \mathrm{~dB}$ & $-14,8 \mathrm{~dB}$ \\
$1500 \cup \min ^{-1}$ & 11 & $\left|i_{\alpha \beta}\right|=1$ & $-0,8 \mathrm{~dB}$ & $-7,0 \mathrm{~dB}$ \\
$2250 \cup$ min $^{-1}$ & 13 & $\left|i_{\alpha \beta}\right|=1$ & $-1,5 \mathrm{~dB}$ & $-8,1 \mathrm{~dB}$ \\
& 11 & $\left|i_{\alpha \beta}\right|=1$ & $-1,0 \mathrm{~dB}$ & $-4,2 \mathrm{~dB}$ \\
$3000 \mathrm{U} \mathrm{min}^{-1}$ & 13 & $\left|i_{\alpha \beta}\right|=1$ & $-0,7 \mathrm{~dB}$ & $-4,8 \mathrm{~dB}$ \\
& 11 & $\left|i_{\alpha \beta}\right|=1$ & $-1,0 \mathrm{~dB}$ & $-2,6 \mathrm{~dB}$ \\
\hline
\end{tabular}
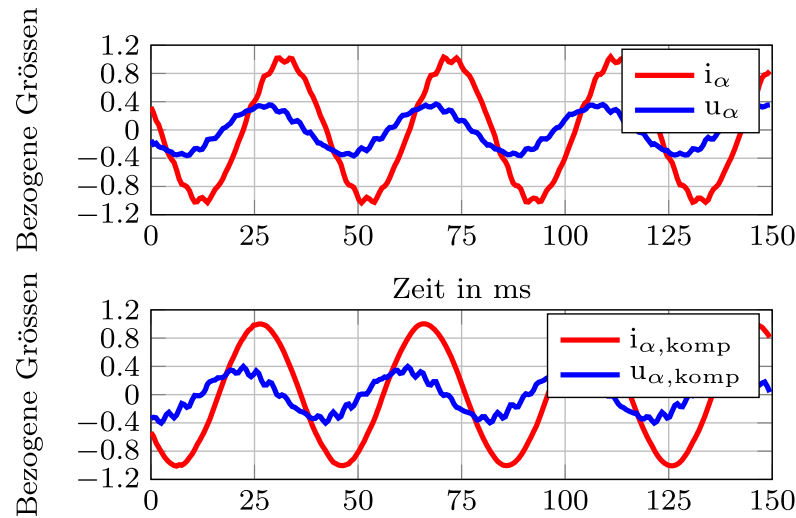

Abb. 12. Stromrippel im Strom $i_{\alpha}$ ohne Kompensation (oben) und mit Kompensation (unten)

ausfiltert. Die so bestimmte reine Oberschwingung wird vom Messsignal wieder abgezogen, wodurch die Messung "blind" bezüglich dieser Frequenz gemacht wird. Durch das Ausblenden der k-ten Harmonischen reagiert der Stromregler nicht auf den Rippel, wodurch auch keine entsprechende Soll-Spannungkomponente $u_{d, \text { soll }}$ erzeugt wird.

Bezüglich der Stabilitäts-Analyse wird ebenfalls das System linearisiert und die Stabilität mittels des Dämpfungsgrads $\xi_{d}$ bzw. $\xi_{q}$ bewertet (siehe Abb. 13). Der optimale Parametersatz für eine robuste Spannungsrippel-Reduktion ergibt sich somit zu (7.1).

$$
\begin{aligned}
& \alpha_{\text {Spannung }}=342^{\circ} \\
& \varepsilon_{\text {Spannung }} \propto \omega_{m}
\end{aligned}
$$

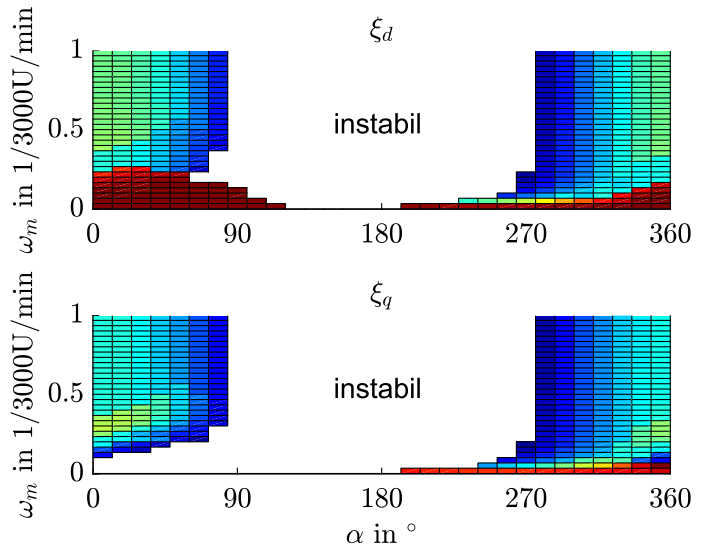

Abb. 13. Spannungsrippel Reduktion: Dämpfungsgrad $\xi_{d}$ und $\xi_{q}$ als Funktion von $\alpha$ und der bezogenen Drehzahl $\omega_{m}$

Abbildung 14 veranschaulicht die Reduktion des Spannungsrippels, wobei die Tab. 2 die gemessene Reduktion des Spannungsrippels bei unterschiedlichen Drehzahlen auflistet. Auch hier sind die Auswirkungen des Kompensationsalgorithmuses auf die anderen verbleibenden Harmonischen im Spannungsrippel vernachlässigbar.

Durch die Reduktion des Rippels in der Strangspannung kann man näher an die Spannungsgrenze des Antriebssystems heranrücken, wodurch der Feldschwäch-Bereich erweitert wird.

\section{Zusammenfassung}

Der Einsatz von Einzelpol-SynRM birgt neben den vielen erwähnten Vorteilen den Nachteil, dass vermehrt unerwünschte Harmonische 


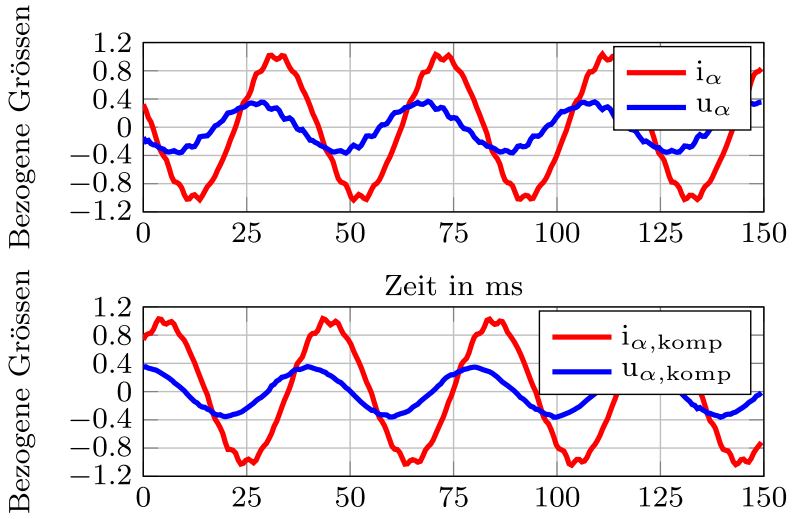

Abb. 14. Spannungsrippel in der Spannung $u_{\alpha}$ ohne Kompensation (oben) und mit Kompensation (unten)

im Strom als auch in der Spannung angeregt werden. Durch das vorgestellte modifizierte mathematische Modell kann das Auftreten der Harmonischen beschrieben und eine Reglerstruktur gefunden werden, welche durch minimales notwendiges Systemwissen die Harmonischen dämpfen bzw. herausregeln kann. Die Reglerstrukturen wurden sowohl für die Unterdrückung des Stromrippels, als auch für die Spannungsrippel-Unterdrückung ausgelegt, am Prototyp getestet und die Performance verifiziert. In zukünftigen Untersuchungen werden die Auswirkungen auf den Wirkungsgrad bzw. auf die Erweiterung des Feldschwächbereichs quantifiziert. Weiters wird dieses Konzept auf die Reduktion von Rippel im Drehmomenten bzw. in der Wirkleistung erweitert. Außerdem soll die Reduktion mehrerer Harmonischer im Sinne der Modalen Regelung untersucht werden.

\section{Danksagung}

Open access funding provided by TU Wien (TUW)

Hinweis des Verlags Der Verlag bleibt in Hinblick auf geografische Zuordnungen und Gebietsbezeichnungen in veröffentlichten Karten und Institutsadressen neutral.

Open Access This article is distributed under the terms of the Creative Commons Attribution 4.0 International License (http://creativecommons.org/ licenses/by/4.0/), which permits unrestricted use, distribution, and reproduction in any medium, provided you give appropriate credit to the original author(s) and the source, provide a link to the Creative Commons license, and indicate if changes were made.

\section{Literatur}

1. Boglietti, A., Pastorelli, M. (2008): Induction and synchronous reluctance motors comparison. In 2008 34th Annual Conference of IEEE Industrial Electronics (S. 20412044). https://doi.org/10.1109/IECON.2008.4758270.
2. Calvo, E. S., Potoradi, D. (2015): Synchronous reluctance motors with and without permanent magnets for high performance low cost electrical drives. In 2015 5th International Electric Drives Production Conference (EDPC) (S. 1-7). https://doi.org/ 10.1109/EDPC.2015.7323220.

3. Guedelhoefer, J., Strbac, D., Gottkehaskamp, R. (2015): Optimization of synchronous reluctance rotors as replacements for squirrel cages of small induction motors up to an iec frame of 90. In IKMT 2015; 10. ETG/GMM-Symposium Innovative small Drives and Micro-Motor Systems (S. 1-6).

4. Hofer, M., Nikowitz, M., Schrödl, M. (2018): Auslegung einer einzelpol-synchronreluktanzmaschine hinsichtlich sensorlosen betriebs eines industriellen antriebs. E\&l, Elektrotech. Inf.tech., 135(2), 153-160. https://doi.org/10.1007/s00502-0180611-X.

5. Hofer, M., Nikowitz, M., Schroedl, M. (2017): Application of a position sensorless control to a reluctance synchronous drive including flux weakening. In PCIM Europe 2017; International Exhibition and Conference for Power Electronics, Intelligent Motion, Renewable Energy and Energy Management (S. 1-7).

6. Hofer, M., Nikowitz, M., Schroedl, M. (2017): Sensorless control of a reluctance synchronous machine in the whole speed range without voltage pulse injections. In 2017 IEEE 3rd International Future Energy Electronics Conference and ECCE Asia (IFEEC 2017 - ECCE Asia) (S. 1194-1198). https://doi.org/10.1109//FEEC.2017.7992211.

7. Hofer, M., Nikowitz, M., Schroedl, M. (2018): Power and efficiency of salient pole and flux barrier type synchronous reluctance machines in low power drives. In 2018 20th European Conference on Power Electronics and Applications (EPE'18 ECCE Europe) (S. 1-6).

8. Hofer, M., Schroedl, M. (2017): Comparison of a flux barrier and a salient pole synchronous reluctance machine for high rotational speeds in electric traction applications. In 2017 20th International Conference on Electrical Machines and Systems (ICEMS) (S. 1-6). https://doi.org/10.1109//CEMS.2017.8056108.

9. Hutterer, M., Kalteis, G., Schrödl, M. (2017): Redundant unbalance compensation of an active magnetic bearing system. Mech. Syst. Signal Process., 94, 267-278. https:// doi.org/10.1016/j.ymssp.2017.02.040.

10. Kalaivani, L., Marimuthu, N. S., Subburaj, P. (2011): Intelligent control for torqueripple minimization in switched reluctance motor. In 2011 1st International Conference on Electrical Energy Systems (S. 182-186). https://doi.org/10.1109/ICEES.2011. 5725325

11. Kazakbaev, V., Prakht, V., Dmitrievskii, V., Sarapulov, S., Askerov, D. (2017): Comparison of power consumption of synchronous reluctance and induction motor drives in a $0.75 \mathrm{~kW}$ pump unit. In 2017 International Siberian Conference on Control and Communications (SIBCON) (S. 1-6). https://doi.org/10.1109/SIBCON.2017.7998485.

12. Masisi, L., Pillay, P., Williamson, S. S. (2016): A modulation strategy for a three-level inverter synchronous reluctance motor (synrm) drive. IEEE Trans. Ind. Appl., 52(2), 1874-1881. https://doi.org/10.1109/TIA.2015.2497307.

13. Nikowitz, M., Hofer, M., Schroedl, M. (2018): Improvements on a sensorless controlled synchronous reluctance machine down to standstill. In PCIM Europe 2018; International Exhibition and Conference for Power Electronics, Intelligent Motion, Renewable Energy and Energy Management (S. 1-7).

14. Shirahase, M., Morimoto, S., Sanada, M. (2010): Torque ripple reduction of srm by optimization of current reference. In The 2010 International Power Electronics Conference - ECCE ASIA (S. 2501-2507). https://doi.org/10.1109/IPEC.2010.5542376.

15. Wu, H., Depernet, D., Lanfranchi, V. (2017): Comparison of torque ripple reductions and copper losses of three synchronous reluctance machines. In 2017 IEEE Vehicle Power and Propulsion Conference (VPPC) (S. 1-6). https://doi.org/10.1109/ VPPC.2017.8330878.

16. Zhong, Z., Jiang, S., Zhou, Y., Zhou, S. (2017): Active torque ripple reduction based on an analytical model of torque. IET Electr. Power Appl., 11(3), 331-341. https:// doi.org/10.1049/iet-epa.2016.0475.

17. Zimmermann, M., Piepenbreier, B. (2017): Design of a permanent magnet assisted synchronous reluctance machine enhanced for saliency based sensorless control. In 2017 IEEE International Electric Machines and Drives Conference (IEMDC) (S. 1-7). https://doi.org/10.1109/IEMDC.2017.8002018. 


\section{Autoren}

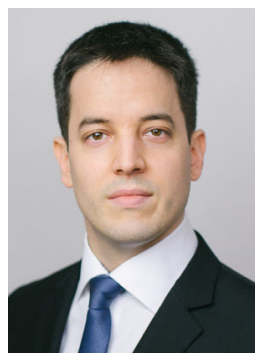

\section{Mario Nikowitz}

Studium der Elektrotechnik an der Technischen Universität Wien, Österreich, Studienzweig Automatisierungstechnik; Abschluss 2016. Seit 2016 Universitätsassistent am Institut für Energiesysteme und Elektrische Antriebe an der TU Wien.

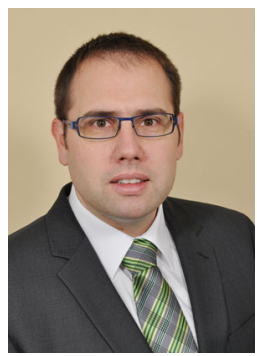

\section{Matthias Hofer}

Studium der Elektrotechnik an der Technischen Universität Wien, Österreich, Studienzweig Automatisierungs- und Regelungstechnik; Abschluss 2004. Universitäts- und Projektassistent am Institut für Elektrische Maschinen und Antriebe der TU Wien 2004 bis 2009, Projektleiter und Abteilungsleiter für die Berechnung elektrischer Maschinen bei Magna Electronics und Magna Powertrain
2009 bis 2013, 2013 Promotion zum Dr. techn., seit 2013 Universitätsassistent am Institut für Energiesysteme und Elektrische Antriebe an der TU Wien.

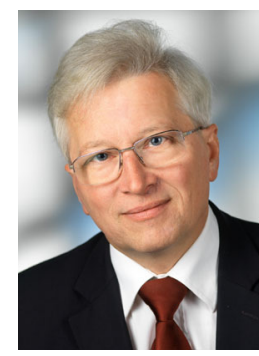

\section{Manfred Schrödl}

Studium der Elektrotechnik an der Technischen Universität Wien, Österreich, Studienzweig Industrielle Elektronik und Regelungstechnik; Abschluss 1982. Universitätsassistent am Institut für Elektrische Maschinen und Antriebe der TU Wien, 1987 Promotion zum Dr. techn., 1992 Habilitation für Elektrische Antriebe und Leistungselektronik in der Antriebstechnik. 1993 bis 1996 Leiter F\&E bei Elin Verkehrstechnik Wien, 1996 bis 1998 Bereichsleiter Zentrale Technik bei Flender ATB, Spielberg, Steiermark. Seit 1998 Ordentlicher Universitätsprofessor für Elektrische Antriebe und Maschinen sowie Vorstand des Instituts für Energiesysteme und Elektrische Antriebe an der TU Wien. 